\title{
AVALIAÇÃO DA POTENCIALIDADE DE ADSORÇÃO DA BUCHA VEGETAL (Luffa cylindrica) PARA REMOÇÃO DE CORANTE DE MEIOS AQUOSOS
}

\author{
Angela Antunes, ${ }^{1,2}$, Francine Todescato de Mello ${ }^{2}$, Cézar Augusto de Aguiar Arpini ${ }^{2}$, Janaína Piasson $^{2}$, Aline Matuella Moreira \\ Ficanha $^{1}$, Luciana Dornelles Venquiaruto ${ }^{2}$ e Rogério Marcos Dallago ${ }^{1,2}$ \\ ${ }^{I}$ Departamento de Engenharia de Alimentos, Universidade Regional Integrada do Alto Uruguai e das Missões, 99700-000, Erechim, \\ Brasil \\ ${ }^{2}$ Departamento de Química, Universidade Regional Integrada do Alto Uruguai e das Missões, 99700-000, Erechim, Brasil
}

*E-mail: nenaantunes@gmail.com

Recebido em: 03/08/2015 Aceito em: 22/02/2016

\section{RESUMO}

A adsorção é um dos processos mais utilizados na remoção de corantes devido à facilidade de manuseio, baixo custo e alta eficiência. Neste sentido, há uma demanda por materiais renováveis para este processo. Neste trabalho, avaliou-se a capacidade adsortiva da bucha vegetal (Luffa cylindrica) e como molécula modelo foi empregado o azul marinho direto. Inicialmente, a bucha vegetal foi ativada com $\mathrm{HCl}$ e NaOH. A amostra ativada com ácido clorídrico apresentou um melhor resultado para a adsorção, de vido a suas propriedades físico-químicas. Posteriormente, o estudo foi conduzido empregando um planejamento estatístico $2^{2}$, variando tempo de contato e concentração do ácido. A análise estatística dos resultados permitiu propor um modelo, o qual foi validado a partir da análise de variância. Das variáveis estudadas, o tempo de contato (6 horas) foi o que apresentou efeito significativo. Verificada a melhor condição para ativação da amostra, partiu-se para um planejamento estatístico $2^{2}$, variando $\mathrm{pH}$ da solução e temperatura. Os resultados mostraram que o acerto do $\mathrm{pH}$ com solução de $\mathrm{HCl} 0,05 \mathrm{~mol} / \mathrm{L}^{-1}$ e temperatura de $45^{\circ} \mathrm{C}$ foram as condições que apresentaram maior capacidade adsortiva. Com esse trabalho, demonstrou-se a viabilidade técnica para a adsorção de corante utilizando bucha vegetal como adsorvente.

Palavras-chave: Bucha Vegetal. Capacidade Adsortiva. Meios Aquosos. Corantes.

\section{Introdução}

O crescente desenvolvimento industrial observado nas últimas décadas, necessário para satisfazer às demandas geradas pelo desmedido aumento populacional, traz consigo sérios problemas ambientais, consequências da produção e, principalmente, do descarte inadequado de seus resíduos. Dentro deste contexto, o setor têxtil caracteriza-se por gerar grandes quantidades de despejos altamente poluidores, os quais contêm elevada carga orgânica e, principalmente, cor acentuada. Estima-se que $20 \%$ dos corantes consumidos durante $\mathrm{o}$ processo de tingimento, são descartados como efluentes, devido à sua incompleta fixação à fibra têxtil [1-3].
Como utiliza um volume substancial de água, a indústria têxtil caracteriza-se como uma das principais fontes de poluição, despejando toneladas de rejeitos industriais contaminados, principalmente, por corantes [4]. Os corantes são substâncias, normalmente, aplicáveis em soluções e que se fixam a um substrato. São, em geral, moléculas pequenas que contêm dois componentes principais: o cromóforo, responsável pela cor e o grupo funcional, que liga o corante à fibra, e devem ser estáveis à luz e aos processos de lavagem [5,6].

A maioria dos corantes, comercialmente, usados é resistente à luz, biodegradação, à fotodegradação e à ação de agentes oxidantes [7]. Esses resíduos coloridos são prejudiciais para a vida aquática onde são descarregadas.[8,9] Os corantes 
são altamente tóxicos para alguns organismos e, portanto, perturbam o ecossistema $[10,11]$.

O corante azul marinho direto pertence ao grupo dos corantes azoicos onde sua estrutura química é formada por um ou mais grupamento azo $(-\mathrm{N}=\mathrm{N}-)$, ligados a sistemas aromáticos (Figura 1). São obtidos no interior das fibras, no momento da reação de tingimento, a partir da aplicação de dois componentes, um de cada vez:alfa naftol e uma base. O alfa naftol é um composto aromático insolúvel em água, mas que pela solubilização com hidróxido de sódio, torna-se um naftolato, que monta (fixa) na celulose. A ligação do naftol com a base produz o corante através de um processo que se denomina copulação. É o mais utilizado e $60 \%$ a $70 \%$ dos corantes podem ser classificados na categoria de corantes azo [12].

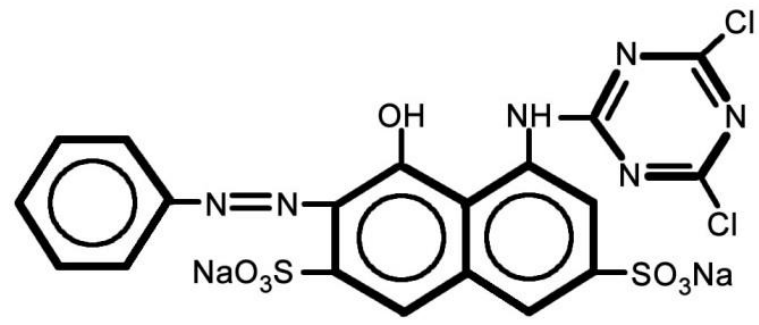

Figura 1. Estrutura química do corante AZO RR2[13]

Do ponto de vista ambiental, a remoção da cor dos efluentes é um dos grandes problemas enfrentados pelo setor têxtil. A elevada estabilidade biológica dos corantes dificulta sua degradação pelos sistemas de tratamento convencionais, baseados em processos físico-químicos (coagulação química) e biológicos (lodos ativados), sendo descartados nos corpos receptores, sem tratamento prévio adequado. Os corantes, quando presentes em rios e lagos, devido às suas intensas colorações, restringem a passagem de radiação solar. A ausência de luz natural provoca uma diminuição na atividade fotossintética natural, provocando alterações na biota aquática, causando toxicidade aguda e crônica nesses ecossistemas [14$18]$.

Geralmente, a poluição é caracterizada pela alta carência de oxigênio e demanda química e bioquímica de oxigênio (DQO e DBO), sólidos suspensos, mau cheiro, toxicidade (presença de compostos contendo enxofre, fenóis, metais pesados acima dos limites estabelecidos pela legislação[19]), e, especialmente, cor. A cor é o primeiro contaminante a ser reconhecido nas águas residuais e sua presença é indesejável, pois, em muitos casos de poluição orgânica, os problemas comodores são percebidos mais facilamente. [20-22].

Buscando maximizar a remoção de cor deste tipo de efluente, diferentes processos alternativos estão sendo avaliados independentemente ou associados aos tratamentos convencionais.

Os processos físicos, como a adsorção em materiais sólidos, possuem maior aplicação industrial, pois associam baixo custo a elevadas taxas de remoção. Além disso, por não se tratar de um método destrutivo, a adsorção, possibilita em alguns casos, a recuperação do corante sem perda de sua identidade química [23,24].

Atualmente, o material que apresenta maior capacidade de adsorção, sendo amplamente utilizado para o tratamento de efluentes, é o carvão ativado de origem vegetal. No entanto, em função de sua superfície ser quimicamente positiva, a adsorção de corantes de caráter catiônico é limitada [25].

Existe um crescente interesse na busca de materiais alternativos de baixo custo que possam ser utilizados, em substituição ao carvão ativado, como adsorventes para a eliminação de corantes têxteis, tais como: argilas ativadas [20], bagaço de cana [26], algas marinhas [27], madeira [28], e outros materiais celulósicos [29].

A bucha vegetal (Figura 2) apresenta baixa densidade, alta porosidade, volume específico dos poros bastante alto, estabilidade em uma ampla faixa de $\mathrm{pH}$ e resistência a autoclavagens sem a ocorrência de danos, mudanças de formas, estrutura e textura, além de estar dotada de um intrincado conjunto de fibras finas lignocelulósicas hidrofílicas, resistentes, elásticas e suaves [30]. A bucha vegetal pode ser encontrada comercialmente nas formas natural e tingida, indicando capacidade de interação com corantes.

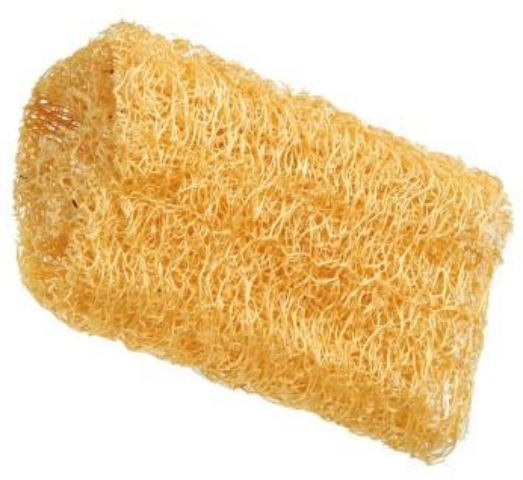

Figura 2. Bucha vegetal (Luffa cylindrica)

Neste contexto, este trabalho teve como objetivo investigar a capacidade adsortiva da bucha vegetal (Luffa cylindrica) na remoção de corante em efluentes aquosos. Para os ensaios adsortivos, conduzidos através de planejamento experimental, foi empregada como molécula modelo o corante azul marinho 
direto. Os dados foram ajustados ao modelo de Langmuir e foram calculados os parâmetros termodinâmicos do processo de interação corante/bucha na interface sólido/líquido.

\section{Parte Experimental ou Metodologia}

\subsection{Material}

A esponja vegetal Luffa cylindrica, empregada neste estudo, foi colhida no interior do município de Erechim/RS. Após coletada, a amostra foi seca em estufa a $105^{\circ} \mathrm{C}$ por $24 \mathrm{~h}$, triturada em um moinho de facas e acondicionada em recipiente vedado, para efeito da reprodutibilidade dos resultados, os experimentos foram realizados em triplicata.

\subsection{Caracterização}

A amostra foi caracterizada quanto a sua área superficial e seu ponto de carga isoelétrico. A área superficial foi determinada mediante análise de adsorção de nitrogênio, empregando o aparelho da marca Quantachrome, modelo Nova 2200 e. Os experimentos foram conduzidos isotermicamente a $196^{\circ} \mathrm{C}$. A amostra $(100 \mathrm{mg})$ foi submetida a uma etapa de desidratação a vácuo por aproximadamente 35 minutos, a temperatura ambiente. Os valores de área superficial foram calculados, conforme o método descrito por Brunauer-EmmettTeller (BET) [31].

\subsection{Determinação do ponto isoelétrico}

Para a determinação do ponto isoelétrico foram pesadas exatamente $0,1 \mathrm{~g}$ de esponja em 18 frascos de $50 \mathrm{~mL}$. Em 9 frascos foram adicionados $50 \mathrm{~mL}$ da solução padrão de $\mathrm{NaCl} 0,1 \mathrm{~mol} \mathrm{~L}^{-1}$. Nos outros 9 frascos adicionou-se $50 \mathrm{~mL}$ da solução padrão de $0,1 \mathrm{~mol} \mathrm{~L}^{-1}$. Os recipientes foram bem vedados para evitar a entrada de $\mathrm{CO}_{2}$ e evaporação. Posteriormente, os $\mathrm{pHs}$ de cada frasco foram ajustados aos valores desejados $(2,5 ; 4,0 ; 5,0 ; 6,0 ; 7,0 ; 8,0 ; 9,0 ; 10,0 ; 11)$ mediante a adição de $\mathrm{HCl}$ e $\mathrm{NaOH} 0,1 \mathrm{~mol} \mathrm{~L}^{-1}$. A variação do $\mathrm{pH}$ foi acompanhada com o auxílio de um pHmetro (modelo UP-25, marca Denver Instruments). Após agitação constante por 24 horas a $25^{\circ} \mathrm{C}$, as amostras foram filtradas (filtração rápida) e o $\mathrm{pH}$ medido novamente.

\subsection{Ensaios de ativação}

Preliminarmente foram realizados ensaios de adsorção, empregando a bucha vegetal submetida a distintas condições de ativação: em $\mathrm{NaOH}$ e em $\mathrm{HCl} 0,1 \mathrm{~mol} \mathrm{~L}^{-1}$. Para estes ensaios, conduzidos sem agitação, 0,5 g de adsorvente foram deixados em contato com $50 \mathrm{~mL}$ de ambas soluções por um período de 24 horas. Posteriormente, as amostras foram filtradas e secas em estufa a $105^{\circ} \mathrm{C}$ por 24 horas. Os ensaios de adsorção foram conduzidos, empregando $0,1 \mathrm{~g}$ de adsorvente e $20 \mathrm{~mL}$ de uma solução do corante $500 \mathrm{mg} \mathrm{L}^{-1}$. De posse dos resultados destes ensaios, foi realizado um planejamento experimental, variando o tempo de contato e a concentração da solução ativadora $(\mathrm{HCl})$, visando a otimização do processo de ativação.

\subsection{Testes de Adsorção}

Para determinar o tempo de contato entre adsorvente e adsorbato, foram realizados ensaios em batelada, a temperatura ambiente $\left(25^{\circ} \mathrm{C}\right)$, empregando $20 \mathrm{~mL}$ dos respectivos corantes (100 $\left.\mathrm{mg} \mathrm{L}^{-1}\right)$ e $0,1 \mathrm{~g}$ de bucha vegetal. Para cada período prédeterminado (10, 30, 60, 180, 360, 720 e 1440 minutos,) foi conduzido um ensaio independente. A quantidade adsorvida foi determinada mediante análise das soluções resultantes, por espectrofotometria UV-Vis (Agilent, modelo 8453), em 582 $\mathrm{nm}$.

A capacidade adsortiva foi avaliada, empregando um planejamento experimental, considerando a influência dos fatores temperatura e $\mathrm{pH}$, mantendo-se constante a quantidade de amostra utilizada $(0,1 \mathrm{~g})$, agitação $(50 \mathrm{rpm})$ e o volume de solução $500 \mathrm{mg} \mathrm{L}^{-1}(20 \mathrm{~mL})$.

Para a obtenção das isotermas de adsorção, as soluções, nas concentrações de 10, 50, 100, 250 e $500 \mathrm{mg} \mathrm{L}^{-1}$, foram deixadas em contato com o material adsorvente $(0,1 \mathrm{~g})$, sob agitação mecânica, a temperatura ambiente, por um período de 24 horas.

\section{Resultados e discussões}

\subsection{Caracterização e ponto isoelétrico}

A bucha vegetal apresentou uma baixa área superficial, de $2,21 \mathrm{~m}^{2} / \mathrm{g}$. A amostra avaliada, neste estudo, apresentou um ponto isoelétrico em $\mathrm{pH}$ 6, como pode ser observado na Figura 3, ou seja, abaixo deste pH, ela apresentará superfície carregada positivamente e acima, negativamente.

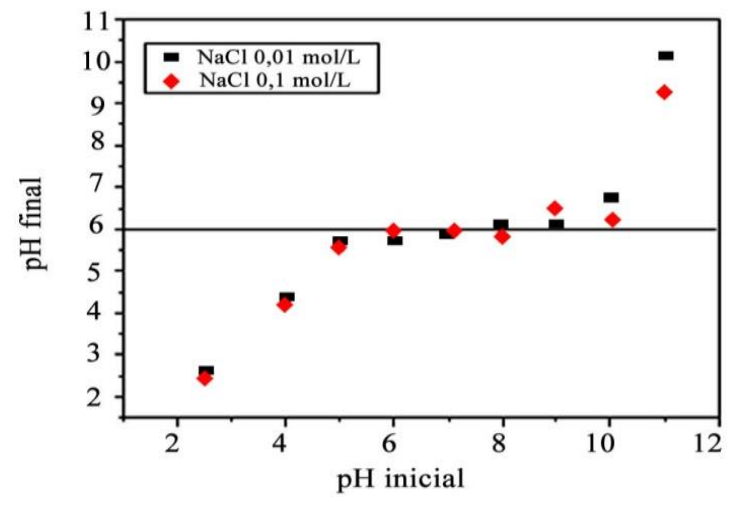

Figura 3. Ponto isoelétrico da bucha vegetal 


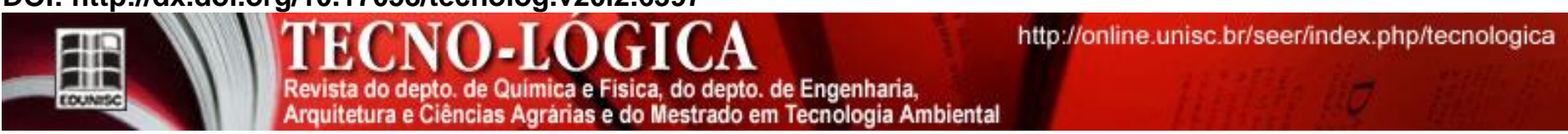

$\mathrm{O}$ pH afeta a densidade e a distribuição de cargas elétricas na molécula de sólidos de tal forma que em determinado $\mathrm{pH}$ o sólido apresentará um mínimo de solubilidade (ponto isoelétrico). No ponto isoelétrico, o número de cargas positivas e negativas é igual, portanto, elas se neutralizam intramolecularmente. Dependendo do $\mathrm{pH}$, há uma maior interação entre solvente e adsorvente. $\mathrm{O}$ ponto isoelétrico varia de produto para produto (sólido) [32].

\subsection{Ensaios de adsorção}

Os ensaios em relação ao tempo de equilíbrio demonstraram que o mesmo é atingido com aproximadamente 90 minutos de contato, conforme pode ser verificado na Figura 4. Com o tempo de contato pré-estabelecido foram realizados ensaios com diferentes processos de ativação.

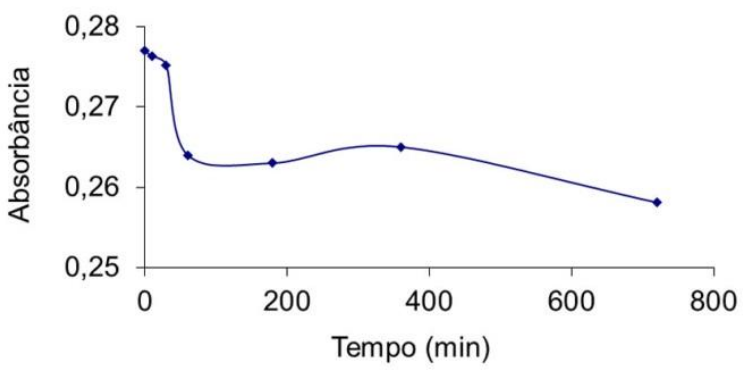

Figura 4. Tempo de equilíbrio para o corante Azul Marinho Direto

Os resultados da quantidade adsorvida estão apresentados na Tabela 1.

Tabela 1. Quantidade adsorvida de corante pela bucha vegetal ativada com $\mathrm{HCl}$ e $\mathrm{NaOH}$

\begin{tabular}{cc}
\hline Amostra & $\mathbf{q}_{\mathrm{eq}}\left(\mathbf{m g g}^{-1}\right)$ \\
\hline Ativação com $\mathbf{~ N a O H}$ & 15,67 \\
Ativação com HCl & 24,04 \\
Natural & 17,14 \\
\hline
\end{tabular}

Para o corante azul marinho direto, de características aniônicas, observou-se um aumento da quantidade adsorvida para o ensaio conduzido com a amostra ativada em meio ácido. Os resultados obtidos estão coerentes com as propriedades físico-químicas do adsorvente e do corante. $\mathrm{Em} \mathrm{pH} 4,0$, o adsorvente encontra-se abaixo do seu ponto de carga isoelétrico, induzindo a geração de sítios positivos em sua superfície. Estes são os sítios responsáveis pela interação, de caráter eletrostático, com o corante de características aniônicas.

Considerando a ativação ácida, um planejamento experimental foi realizado para a otimização desta etapa, variando-se o tempo de contato e a concentração de $\mathrm{HCl}$ ( mol $\left.\mathrm{L}^{-1}\right)$. Os níveis e respostas em termos da quantidade de corante adsorvido por grama de adsorvente são apresentados na Tabela 2.

Tabela 2. Matriz do planejamento $2^{2}$ (valores codificados e reais) com a resposta da quantidade adsorvida

\begin{tabular}{cccc}
\hline Ensaio & $\begin{array}{c}\text { Tempo de } \\
\text { contato (h) }\end{array}$ & $\begin{array}{c}\text { Concentração } \\
\text { de HCl (mol L L }\end{array}$ & $\mathbf{q}_{\mathbf{e q}}\left(\mathbf{m g ~ g}^{-\mathbf{1}}\right)$ \\
\hline $\mathbf{1}$ & $-1(1)$ & $-1(0,05)$ & 11,99 \\
$\mathbf{2}$ & $1(6)$ & $-1(0,05)$ & 20,36 \\
$\mathbf{3}$ & $-1(1)$ & $1(0,15)$ & 10,61 \\
$\mathbf{4}$ & $1(6)$ & $1(0,15)$ & 18,89 \\
5 & $0(3,5)$ & $0(0,1)$ & 12,11 \\
6 & $0(3,5)$ & $0(0,1)$ & 13,77 \\
7 & $0(3,5)$ & $0(0,1)$ & 13,47 \\
\hline
\end{tabular}

Ambas as variáveis avaliadas apresentaram efeitos em relação à quantidade adsorvida. As variações mais expressivas foram observadas para o tempo de contato. $\mathrm{O}$ aumento da quantidade adsorvida, em função do tempo de contato, sugere um efeito positivo para esta variável. Em relação à concentração, observa-se uma diminuição da quantidade adsorvida com o aumento da concentração, sugerindo um efeito negativo desta variável.

A análise estatística apresentada na Tabela 3, confirma os efeitos positivo para o tempo de contato e negativo para a concentração. No entanto, a única variável que foi estatisticamente significativa com $95 \%$ de confiança $(p \leq 0,05)$ foi o tempo de contato. A maior quantidade adsorvida foi de $20,36 \mathrm{mg} \mathrm{g}^{-1}$.

Tabela 1. Coeficientes de regressão para a resposta de quantidade adsorvida $\left(\mathrm{mg} \mathrm{g}^{-1}\right)$

\begin{tabular}{ccccc}
\hline & $\begin{array}{c}\text { Coeficientes } \\
\text { de regressão }\end{array}$ & $\begin{array}{c}\text { Erro } \\
\text { Padrão }\end{array}$ & T & p \\
\hline Média & 14,46 & 0,73 & 19,99 & 0,00 \\
Tempo & 4,16 & 0,96 & 4,35 & 0,02 \\
Concentração & $-0,71$ & 0,96 & $-0,75$ & 0,51 \\
Interação & $-0,02$ & 0,96 & $-0,02$ & 0,98 \\
\hline
\end{tabular}

A partir desses resultados, o modelo empírico de segunda ordem proposto para a quantidade de adsorção no delineamento experimental completo $2^{2}$ é dado pela Equação 1:

$\mathrm{q}_{\mathrm{eq}}\left(\mathrm{mg} \mathrm{g}^{-1}\right)=14,46+4,16 \mathrm{~T}$

A análise de variância possibilitou validar o modelo empírico proposto na Equação 1, uma vez que o $\mathrm{F}_{\text {calculado }}(6,49)$ foi maior que o $F_{\text {tabelado }}(5,39)$, com uma porcentagem de variação explicada de $86 \%$, permitindo a construção da superfície de resposta e da curva de contorno apresentadas na Figura 5. 


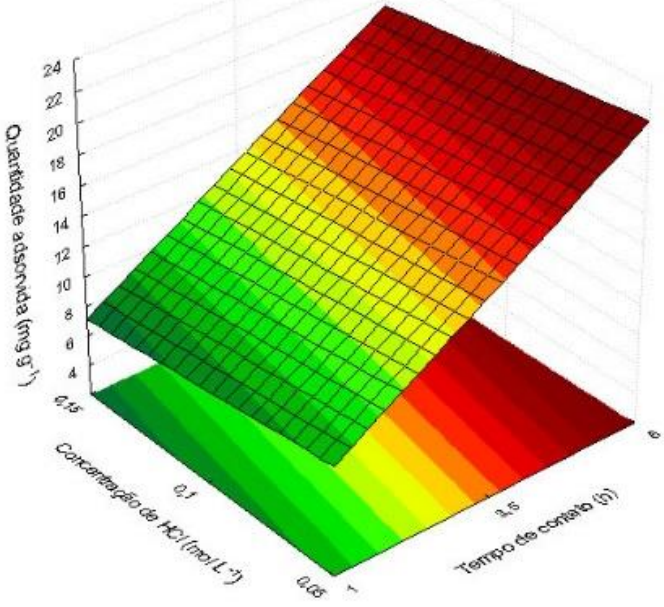

Figura 5. Superfície de resposta e curva de contorno para a adsorção com azul marinho direto.

Ensaios de ativação com tempos de contato mais prolongados (12 e 24 horas) foram realizados. No, entanto os valores de quantidade adsorvida observados foram similares aos observados para 6 horas de contato.

Empregando-se a condição de ativação otimizada $(\mathrm{HCl}$ $0,05 \mathrm{~mol} \mathrm{~L}^{-1}$ e 6 horas de contato), foi realizado um ensaio de otimização das condições de adsorção.

Neste contexto, foi realizado um segundo planejamento $2^{2}$, variando-se $\mathrm{pH}$ e temperatura. A Tabela 4 apresenta as variáveis e níveis estudados e os resultados obtidos, em $\mathrm{mg} \mathrm{g}^{-1}$.

Tabela 2. Matriz do planejamento $2^{2}$ (valores codificados e reais) com a resposta da quantidade adsorvida

\begin{tabular}{cccc}
\hline Ensaio & $\mathbf{p H}$ & $\begin{array}{c}\text { Temperatura } \\
\left({ }^{\mathbf{0}} \mathbf{C}\right)\end{array}$ & $\mathbf{q}_{\text {eq }}\left(\mathbf{m g ~ g}^{-\mathbf{1}}\right)$ \\
\hline $\mathbf{1}$ & $-1(4)$ & $-1(15)$ & 21,37 \\
$\mathbf{2}$ & $1(10)$ & $-1(15)$ & 11,78 \\
$\mathbf{3}$ & $-1(4)$ & $1(45)$ & 33,45 \\
$\mathbf{4}$ & $1(10)$ & $1(45)$ & 11,03 \\
$\mathbf{5}$ & $0(7)$ & $0(30)$ & 26,51 \\
$\mathbf{6}$ & $0(7)$ & $0(30)$ & 25,96 \\
$\mathbf{7}$ & $0(7)$ & $0(30)$ & 26,40 \\
\hline
\end{tabular}

Para este experimento, observa-se uma diminuição da quantidade adsorvida em função do aumento do $\mathrm{pH}$, indicando um efeito negativo para esta variável. Esta tendência foi vinculada a alterações de carga na superfície do adsorvente, o qual apresenta um $\mathrm{pH}$ de ponto de carga zero igual a 6,0. Para pHs maiores que 6,0, a geração de cargas negativas na superfície do adsorvente tende a repelir as moléculas do corante de características aniônicas (negativas), minimizando a quantidade adsorvida.

O aumento da quantidade adsorvida, em função da temperatura, indica um efeito positivo para esta variável. A análise estatística, deste planejamento, mostrou que não foi possível se obter um modelo empírico válido estatisticamente $(\mathrm{p}<0,05)$. Desta forma, os resultados são apresentados na forma da tabela de efeitos, apresentada na Tabela 5.

Tabela 3. Coeficientes de regressão para a resposta de quantidade adsorvida (mg g-1)

\begin{tabular}{ccccc}
\hline & $\begin{array}{c}\text { Coeficientes } \\
\text { de regressão }\end{array}$ & $\begin{array}{c}\text { Erro } \\
\text { Padrão }\end{array}$ & $\mathbf{t}(3)$ & P \\
\hline Média & 22,36 & 0,11 & 204,00 & 0,00024 \\
(1) pH & $-8,00$ & 0,14 & $-55,20$ & 0,000328 \\
(2) Temperatura & 2,83 & 0,14 & 19,54 & 0,002610 \\
$\mathbf{1}$ x 2 & $-3,21$ & 0,14 & $-22,13$ & 0,002035 \\
\hline
\end{tabular}

\subsection{Isotermas de adsorção}

Considerando que a temperatura apresenta um efeito positivo, foram conduzidos ensaios para a determinação da isoterma de adsorção, empregando distintas temperaturas, fixando o pH em 4. As isotermas obtidas são apresentadas na Figura 6. 


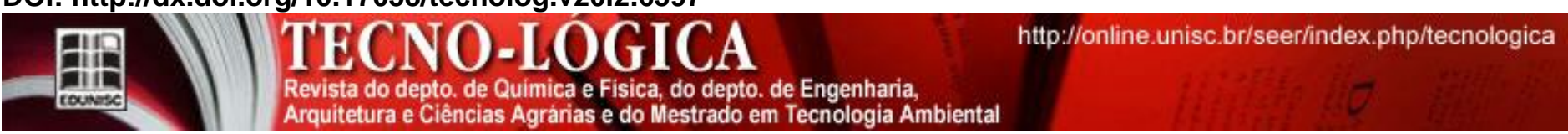

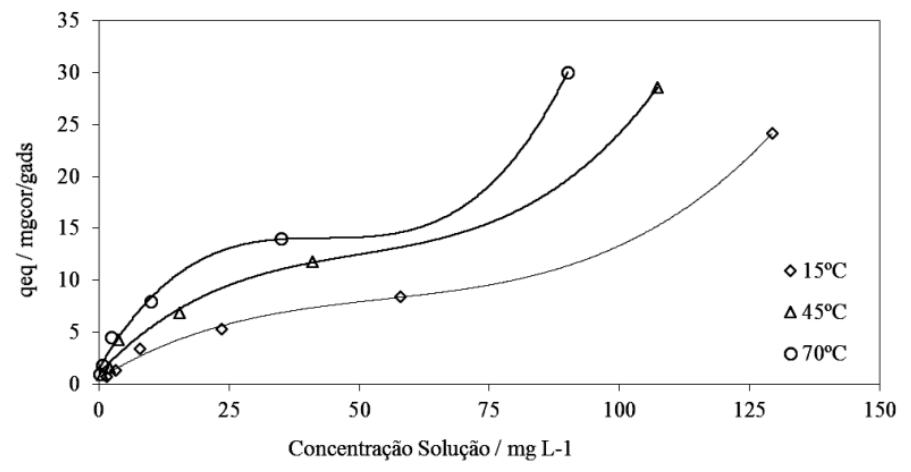

Figura 6. Isoterma de adsorção para o corante Azul Marinho Direto

Observa-se um aumento da quantidade adsorvida em função da temperatura. Os resultados obtidos para as isotermas estão coerentes com os do planejamento experimental, descrito anteriormente, onde o aumento da temperatura favorece a adsorção. A linearização dos dados obtidos, experimentalmente, demonstrou adequação somente para o modelo de Langmuir, conforme observado na Figura 7.

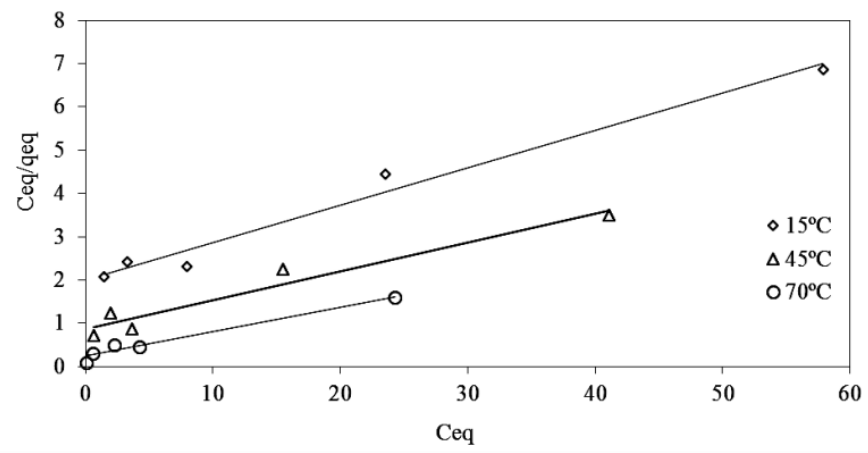

Figura 7. Isoterma de adsorção do corante Azul Marinho Direto ajustada para o modelo de Langmuir

Os valores das constantes de Langmuir ( $\mathrm{K} \mathrm{e} \mathrm{N}$ ), onde $\mathrm{K}$ é a constante de equilíbrio e $\mathrm{N}$ é a capacidade máxima de adsorção do sólido são apresentados na Tabela 6. Pode-se observar que a capacidade adsortiva aumenta com a temperatura. Quanto maiores os valores de N, mais capacidade de adsorção o sólido terá. Considerando os valores para $\mathrm{K}$, observa-se que a espontaneidade do processo aumenta com a temperatura, favorecendo a adsorção. $\mathrm{O}$ valor de $\mathrm{K}$ corresponde se os ensaios realizados estão mais para a adsorção ou dessorção. Quanto maior o valor de K, mais espontânea será a reação, e mais adsorção ocorrerá.
Tabela 6. Constantes de Langmuir e valor de $\mathrm{R}^{2}$ obtidos a partir dos resultados de adsorção de corante em bucha vegetal

\begin{tabular}{cccc}
\hline Temperatura & $\mathbf{K}$ & $\begin{array}{c}\text { Parâmetros } \\
\mathbf{N}\end{array}$ & $\mathbf{R}^{\mathbf{2}}$ \\
\hline $15^{\circ} \mathrm{C}$ & 0,04294 & 11,61 & 0,979 \\
$45^{\circ} \mathrm{C}$ & 0,07586 & 15,06 & 0,945 \\
$70^{\circ} \mathrm{C}$ & 0,23603 & 17,66 & 0,968 \\
\hline
\end{tabular}

O cálculo dos parâmetros termodinâmicos, referente ao processo de adsorção do corante azul marinho direto, foi determinados através da inclinação da reta obtida a partir da regressão linear de $\operatorname{lnK}$ em função de $\mathrm{T}^{-1}$, apresentados na Figura 8.

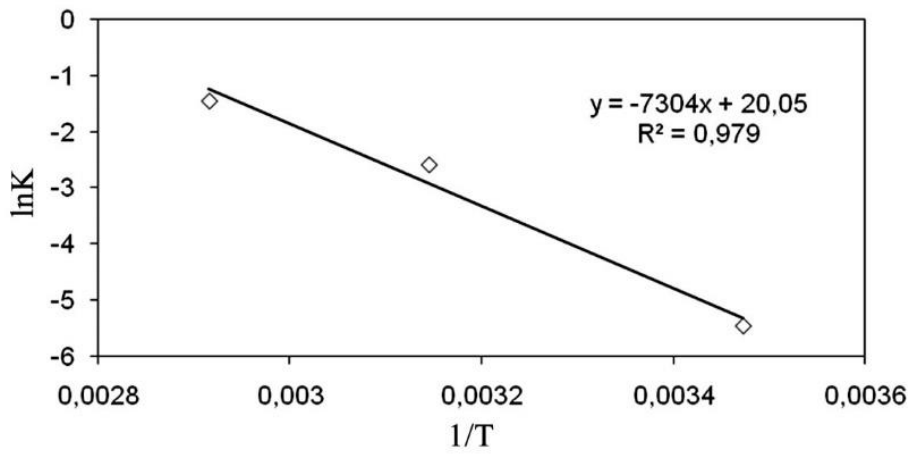

Figura 8. Linearização dos dados de adsorção para o corante azul marinho direto

A partir desta linearização foi possível a obtenção dos parâmetros termodinâmicos. Os resultados obtidos para entalpia, entropia e energia livre de Gibbs são apresentados na Tabela 7.

Tabela 4. Parâmetros termodinâmicos obtidos da adsorção do corante azul marinho direto no adsorvente de bucha vegetal, considerando uma temperatura de $25^{\circ} \mathrm{C}(298 \mathrm{~K})$

\begin{tabular}{ccc}
\hline$\Delta \mathrm{H}\left(\mathrm{kJ} \mathrm{mol}^{-1}\right)$ & $\Delta \mathrm{S}_{\left(\mathrm{J} \mathrm{k}^{-1} \mathrm{~mol}^{-1}\right)}$ & $\Delta \mathrm{G}^{\mathrm{o}}\left(\mathrm{kJ} \mathrm{mol}^{-1}\right)$ \\
\hline$+60,7$ & $+166,7$ & $+11,05$ \\
\hline
\end{tabular}

$\mathrm{O}$ valor de entalpia de adsorção $(\Delta \mathrm{H})$ obtido foi de 60,7 $\mathrm{kJ} \mathrm{mol}^{-1}$. Valores positivos de $\Delta \mathrm{H}$ confirmam a natureza endotérmica do processo de adsorção, enquanto valores positivos

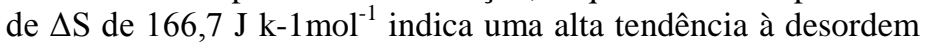
na interface bucha-solução AMD [33].

Valores de $\Delta \mathrm{H}$ menores que $42 \mathrm{~kJ}^{-1} \mathrm{~mol}^{-1}$ indicam um processo de adsorção física [34]. $\mathrm{O} \Delta \mathrm{H}$ obtido no processo foi de $60,7 \mathrm{~kJ} \mathrm{~mol}^{-1}$, indicando um processo de natureza química.

$O$ valor da energia livre $(\Delta G)$, além de medir a força que guia determinada reação, também indica quão distante do equilíbrio está o estado inicial do sistema. $\mathrm{O}$ valor positivo de $\Delta \mathrm{G}$, obtido neste experimento, indica que a forma adsorvida é menos estável que aquela em solução. A variação positiva da entropia indica a afinidade entre adsorvato e a solução do adsorvente (processo não - espontâneo) [35]. 


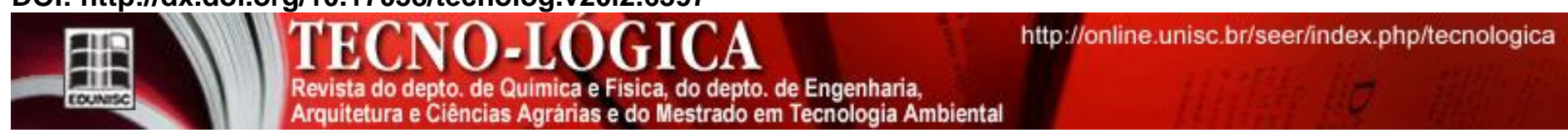

\section{Conclusões}

Com base nos resultados apresentados, utilização de bucha vegetal (Luffa cylindrica) como adsorvente, apresentou boa eficiência na remoção do corante estudado. Dessa forma, a bucha vegetal apresenta-se como uma alternativa econômica e eficiente para a remoção de corantes, devido ao adsorvente ser um material inovador, pois até onde se tem pesquisado não há nada na literatura que utiliza essa esponja vegetal como alternativa para o tratamento de resíduos têxteis. O efeito da ativação observado para este adsorvente foi vinculado ao seu ponto de carga zero (pH 6,0). Os resultados de ativação demonstraram um efeito significativo do tempo de contato da etapa de ativação com $\mathrm{HCl}$. Para os ensaios adsortivos, o efeito negativo observado para o $\mathrm{pH}$ está coerente com as propriedades físico-químicas do adsorvente e do corante empregado como molécula modelo. $\mathrm{O}$ efeito positivo da temperatura, observado tanto no planejamento, quanto nas isotermas, está coerente com as características endotérmicas ( $\Delta \mathrm{H}$ positivo) observadas para este processo.

\section{Agradecimentos}

URI-Erechim, FAPERGS, CNPq, SC\&T/RS.

\section{EVALUATION OF THE PLANT SPONGE (LUFFA \\ CYLINDRICA) ADSORPTION POTENTIAL FOR THE REMOVAL OF DYE FROM AQUEOUS MEDIA}

\begin{abstract}
Adsorption is one of the most widely used processes for coloring removal due to easy handling, low cost and high efficiency. Therefore, there is a demand for renewable materials for this process. The aim of this study was to evaluate the adsorptive capacity of plant sponges (Luffa cylindrica), applying directly the navy blue as molecule model. Initially, the plant sponge was activated using $\mathrm{HCl}$ and $\mathrm{NaOH}$. The sample activated with hydrochloric acid showed a better adsorption result due to its physicochemical properties. Subsequently, the study was carried out using a statistical design $2^{2}$, varying the contact time and acid concentration. A model was proposed from the Statistical analysis of the results, which was validated based on the analysis of variance. According to the variables studied, the contact time (6 hours) showed significant effect. After checking the best condition for sample activation, a statistical design $2^{2}$ was performed, varying the $\mathrm{pH}$ and temperature of the solution. The results showed that $\mathrm{pH}$ adjustment with $\mathrm{HCl}$ solution of 0,05 mol. $\mathrm{L}^{-1}$ and a temperature at $45^{\circ} \mathrm{C}$ were the conditions which presented the highest adsorptive capacity. Technical feasibility to dye adsorption, using plant sponge as adsorbent, was shown in this work.
\end{abstract}

Keywords: Adsorption. Renewable materials. Loofah.

\section{Referências}

BARTZ, T.; SILUK, J. C. M.; RIFFEL, E. T.; Uso da troca rápida de ferramentas - TRF - como estratégia de aumento de produtividade em uma rotuladora de garrafas plásticas, Tecno-Lógica, Vol. 16, n. 2, p. 71-77, 2012.

[1] GUARATINI, C. C. I.; ZANONI, M. V. B.; Corantes têxteis, Química nova, Vol. 23, n. 1, p. 71-78, 2000.

[2] ALCÂNTARA, M. R.; DALTIN, D.; A química do processamento têxtil, Química nova, Vol. 19, n. 3, p. 320-330, 1996.

[3] WEBER, E. J.; STICKNEY, V. C.; Hydrolysis kinetics of reactive blue 19vinyl sulfone, Water Research, Vol. 27, n. 1, p. 63-67, 1993.

[4] PONNUSAMI, V.; VIKRAM, S.; SRIVASTAVA, S. N.; Guava (Psidium guajava) leaf powder: novel adsorbent for removal of methylene blue from aqueous solutions, Journal of Hazardous Materials, Vol. 152, n. 1, p. 276-286, 2008.

[5] BONANCÊA, Carlos Eduardo. Estudo espectroscópico de processos de degradação fotoquímica e fotoeletroquímica de corantes. Tese (Programa de PósGraduação em Tecnologia Ambiental - Doutorado) - Universidade de São Paulo. 2010.

[6] BELTRAME, Leocádia Terezinha Cordeiro. Sistemas microemulsionados aplicados à remoção da cor de efluentes têxteis.Tese (Doutorado em Engenharia Química - Doutorado), Universidade Federal do Rio Grande do Norte. 2006.

[7] FUNGARO, D. A.; BRUNO, M.; Utilização de zeólitas sintetizadas a partir de cinza de carvão para remoção de azul de metileno em água, The eletronic Journal of Chemistry, Vol. 1, p. 49-63, 2009.

[8] SHENAI, V. A.; Azo dyes on textiles vs German ban-An objective assessment-Part II Colour Index and German ban on certain azo dyes, Colourage, Vol. 43, p. 33-40, 1996.

[9] GREENE, J. C.; BAUGHMAN, G. L.; Effects of 46 dyes on population growth of fresh green algae Selenastrum capricornutum, Texile Chemist and Color, Vol. 28, p. 4, 1996.

[10] SAHA, C.; Eco-textile: a novel concept of cleaner product, Text Dyer Printer XXIX, Vol. 21, p. 13-16,1996.

[11] PHILLIPS, D. A. S.; Environmentally friendly, productive and reliable priorities for cotton dyes and dyeing processes, Journal Society Dyers Color, Vol. 112, n. 7-8, p. 183-186, 1996.

[12] MALIK, A. S.; Expert Meeting on Environmental Requirements and International Trade, Geneva, Suíca, 2002.

[13] DOS SANTOS, A. B.; Enhancing the electron transfer capacity and subsequent colour removal in bioreactors by applying thermophilic anaerobic treatment and redox mediators, Biotechnology and Bioengineering, Vol. 89, n.1, p. $42-52.2005$.

[14] ZANONI, M. V. B.; CARNEIRO, P. A.; O descarte dos corantes têxteis. Ciência Hoje, Vol. 29, p. 61-64, 2001.

[15] BERTAZZOLI, R.; PELEGRINI, R.; Descoloração e degradação de poluentes orgânicos em soluções aquosas através do processo fotoeletroquímico, Química Nova, Vol. 25, p. 477-482, 2002.

[16] HOUK, V. S.; The genotoxicity of industrial wastes and effluents: a review, Mutation Research Reviews in Genetic Toxicology, Vol. 277, p. 91-138, 1992. 
[17] KNAPP, J. S.; ZHANG, F.; TAPLEY, K. J. N.; Decolourisation of Orange II by a wood-rotting fungus, Journal of Chemical Technology and Biotechnology, Vol. 69, p. 289-296, 1997

[18] KAPDAN, I. K.; KARGI, F.; MCMULLAN, G.; MARCHANT, R.; Biological decolorization of textile dyestuff by Coriolus versicolor in a packed column reactor, Environmental Technology, Vol. 21, p. 231-236, 2000.

[19]Resolução CONAMA No 430/2011 - "Dispõe sobre condições e padrões de lançamento de efluentes, complementa e altera a Resolução no 357, de 17 de março de 2005, do Conselho Nacional do Meio Ambiente - CONAMA." - Data da legislação: 13/05/2011 - Publicação DOU nº 92, de 16/05/2011, pág. 89

[20] CRINI, G.; BADOT, P. M.; Application of chitosan, a natural aminopolysaccharide, for dye removal from aqueous solutions by adsorption processes using batch studies: A review of recent literature, Progress in Polymer Science, Vol. 33, p. 399-447, 2008.

[21] AHMAD, A. A.; HAMEED, B. H.; AZIZ, N.; Adsorption of direct dye on palm ash: Kinetic and equilibrium modeling, Journal of Hazardous Materials, Vol. 141, p. 70-76, 2007.

[22] ARDEJANI, F. D.; BADII, K.; LIMAEE, N. Y.; SHAFAEI, S. Z.; MIRHABIBI, A. R.; Adsorption of Direct Red 80 dye from aqueous solution onto almond shells: Effect of $\mathrm{pH}$, initial concentration and shell type, Journal of Hazardous Materials, Vol. 151, p. 730-737, 2008.

[23] SMITH, B.; KOONCE, T.; HUDSON, S.; Decolorizing dye wastewater using chitosan, American Dyestuff Reporter, Vol. 82, p. 18-35, 1993

[24] OLIVEIRA, L. C. A.; RIOS, R. V.; FABRIS, J. D.; SAPAG, K.; LAGO, R. M.; Activated carbon/iron oxide magnetic composites for the adsorption of contaminants in water, Carbon, Vol. 40, p. 2177-2183, 2002.

[25] AL-DEGS, Y.; KHRAISHEH, M. A. M.; ALLEN, S. J.; AHMAD, M. N.; Effect of carbon surface chemistry on the removal of reactive dyes from textile effluent, Water Research, Vol. 34, p. 927-935, 2000.

[26] OFOMAJA, A. E.; Kinetic study and sorption mechanism of methylene blue and methyl violet onto mansonia (Mansonia altissima) wood sawdust, Chemical Engineering Journal, Vol.143, p. 85-95, 2008.

[27] PAVAN, F. A.; LIMA, E. C.; DIAS, S. L. P.; MAZZOCATO, A. C. J.; Methylene blue biosorption from aqueous solutions by yellow passion fruit waste, Journal of Hazardous Materials, Vol. 150, p. 703-712, 2008.

[28] BESTANI, B.; BENDERDOUCHE, N.; BENSTAALI, B.; BELHAKEM, M.; ADDOU, A.; Methylene blue and iodine adsorption onto an activated desert plant, Bioresource Technology, Vol. 99, n. 17, p. 8441-8444, 2008

[29] DEMIR, H.; TOP, A.; BALKOSE, D.; ULKU, S.; J. Dye adsorption behavior of Luffa cylindrica fibers, Journal of Hazardous Materials, Vol. 153, p. 389-394, 2008.

[30] VICHIATO, M. R. M.; VICHIATO, M.; CASTRO, D. M.; DUTRA, L. F.; PASQUAL, M.; ARAÚJO, T. S.; Bucha vegetal e fertilização organo-mineral no cultivo de Dendrobium nobile lindl, Revista da FZVA, Vol. 15, n. 1, p. 34-42, 2008.

[31] BRUNAUER, S.; EMMET, T. P. H.; TELLER, E.; J.; Surface area measurements of activated carbons, silica gel and other adsorbents, Journal of the American Chemical Society, Vol. 60, p. 309-319, 1938.

[32] CARVALHO, A. V.; GARCÍA, N. H. P.; WADA, J. K. A.; Characterization of a protein concentrate and isolate from cupuassu (Theobroma grandiflorum Schum) seeds, Brazilian Journal Food Technology, Vol. 12, p. 1-8, 2009.

[33] BRUM, S. S.; BIANCHI, M. L.; SILVA, V. L.; GONÇALVES, M.; GUERREIRO, M. C.; OLIVEIRA, L. C.; A Preparação e caracterização de carvão ativado produzido a partir de resíduos do beneficiamento do café, Química Nova, Vol. 31, p. 1048-1052, 2008

[34] GONÇALVES, M.; OLIVEIRA, L. C. A.; GUERREIRO, M. C.; Nióbia magnética como adsorvente de contaminantes orgânicos em meio aquoso: efeito da temperatura e do pH, Química Nova, Vol. 31, p. 518-522, 2008.

[35] GUERRA, D. L.; AIROLDI, C.; LEMOS, V. P.; ANGÉLICA, R. S.; VIANA, R. R.; Aplicação de Zr/Ti-PILC no processo de adsorção de Cu (II), Co (II) e Ni (II) utilizando modelos físico-químicos de adsorção e termodinâmica do processo, Química. Nova, Vol. 31, p. 353-359, 2008. 